\title{
Über das Verhältnis der Theorie der Elementarlänge zur Quantentheorie. II
}

\author{
P. JORDAN
}

Eingegangen am 5. November 1968

\begin{abstract}
The idea put forward in I that also such algebras which do not fulfil the axiom of powerassociativity may allow in some cases physical interpretation, is illustrated by a mathematical model showing properties similar to the Cartesian coordinates of a particle.
\end{abstract}

\section{$\S 1$}

In einer vorangegangenen Note (im folgenden als I bezeichnet) wurde die Anregung vorgetragen, als mathematische Beschreibung: mikrophysikalischer Systeme, die über die Zuständigkeitsgrenzen der Quantenmechanik vielleicht hinausgehen, auch solche Algebren in Betracht zu ziehen, welche nicht mehr potenzassoziativ sind. (Die gewohnte mathematische Formulierung der Quanten-Theorie, gegründet auf die assoziativen Matrix- oder Operator-Algebren, ist - wie in I erläutert wurde - mathematisch im wesentlichen gleichbedeutend mit der $\mathrm{Zu}$ grundelegung solcher Algebren, die kommutativ und potenzassoziativ sind.)

Es wurde in I gezeigt, daß eine Zulassung auch solcher Algebren, die nicht mehr potenzassoziativ sind, grundsätzlich geeignet sein kann, physikalisch interpretierbare mathematische Modelle zu liefern. Ob solche Modelle beitragen können zu einer Beschreibung realer physikalischer Verhältnisse, wird natürlich nur in ausgedehnterer Erprobung entschieden werden können.

Jedoch soll im folgenden durch ein konkretes Beispiel belegt werden, daß die erwogene Erweiterung in Betracht gezogener mathematischer Möglichkeiten unter Umständen recht reizvolle Modelle in den Kreis der Untersuchung treten läßt. Das Folgende beabsichtigt ausschließlich eine Demonstrierung dieser Tatsache; es soll keine Vermutung darüber geäußert werden, ob das zu betrachtende mathematische Modell schon einen wirklichen Fortschritt zur Erfassung mikrophysikalischer Realität anbahnen kann.

\section{$\S 2$}

Eine kartesische Ortskoordinate $x$ für ein Elementarteilchen sei ausgedrückt als Vielfaches der Elementarlänge; danach ist $x$ also dimensionslos. Die Algebra $\mathfrak{A}$ der von $x$ erzeugten Größen soll die (rekursiv definier- 
ten) Potenzen $x^{1}=x ; x^{2}, x^{3}, \ldots, x^{y}=x x^{y-1}$ von $x$ als Basis haben; wir fügen eine Haupteinheit $e=x_{0}$ hinzu. Das kommutative aber nicht assoziative Multiplikationsgesetz der fraglichen Basiselemente sei

$$
x^{\mu} x^{\nu}=\sum_{\varrho=0}^{\infty} \frac{\mu ! \nu ! x^{\mu+\nu-4 \varrho}}{(2 \varrho) !(\mu-2 \varrho) !(\nu-2 \varrho) !} .
$$

Die hingeschriebene Summe ist für jedes Exponentenpaar $\mu, v$ endlich, da alle Glieder mit $2 \varrho>\mu$ oder $2 \varrho>v$ verschwinden.

Insbesondere haben wir also

$$
\left[x, x, x^{2}\right]=2,
$$

(genauer: $=2 e$ ), wenn wir das übliche Assoziator-Zeichen

benutzen.

$$
[a, b, c]=(a b) c-a(b c)
$$

\section{$\S 3$}

$\mathrm{Zu}$ dem Versuch, diese Algebra als diejenige der Funktionen einer (mit Hilfe der Elementarlänge normierten) $x$-Koordinate zu deuten, ermutigen folgende Eigenschaften:

1. Die Formel (1) ist invariant gegen Vorzeichen-Änderung von $x$. Das ist trivial.

2. Die Formel (1) ist invariant gegen Veränderung des Nullpunkts von $x$ : Für

$$
x^{\prime}=x+\xi e
$$

statt $x$, wo $\xi$ eine beliebige Zahl ist, gilt ebenfalls Formel (1).

Den Beweis hierfür erhält man, indem man sich folgendes klar macht:

1. Nach (1) gilt die Beziehung

$$
[x, x u, v]=x[x, u, v]+u[x, x, v]
$$

für beliebige Elementenpaare $u, v$ aus $\hat{\mathfrak{A}}$.

2. Aus (2) und (5) kann (1) als Folgerung abgeleitet werden; Einzelheiten sind in einer Arbeit von JoRdan-Matsushita-RÜHAaK [1] besprochen.

Nun sind aber (2) und (5) invariant gegen die Transformation (4). Folglich ist auch (1) invariant.

\section{$\$ 4$}

In der mathematischen Theorie der nichtassoziativen Algebren [2] arbeitet man viel mit dem Begriff des Multiplikationsoperators $L(u)$ eines Elementes $u$. Die Formel (1) kann damit auch so ausgedrückt werden:

$$
L\left(x^{\mu}\right)=\sum_{\varrho=0}^{\infty}\left(\begin{array}{c}
\mu \\
2 \varrho
\end{array}\right) L(x)^{\mu-2 \varrho} D^{2 \varrho},
$$


wo $D$ der Differentiationsoperator ist, also

$$
[D, L(x)]=1 \text {. }
$$

\section{$\$ 5$}

Wir können, ohne jetzt auf Konvergenzfragen einzugehen, Funktionen von $x$ durch Potenzreihen definieren. Man erhält dann z. B. durch einfache Umformung:

$$
e^{i \alpha x} e^{i \beta x}=e^{i(\alpha+\beta) x} \cos (\alpha \beta),
$$

wo also die gewohnte klassische Formel durch das Auftreten eines Faktors Cosinus hyperbolicus verändert ist.

Diese Formel legt folgende Deutung nahe: In der bisherigen Theorie kann die $x$-Koordinate eines Elektrons (sofern man beliebige Unschärfe des Impulses zuläßt) grundsätzlich mit jeder beliebigen Genauigkeit gemessen werden, da beliebig enge Wellenpakete konstruiert werden können. Nach obiger Formel gilt das jedoch nur noch bei Beschränkung auf solche Wellenlängen, welche groß gegenüber der Elementarlänge sind - werden in der Konstruktion eines Wellenpaketes auch Wellenlängen unterhalb der Elementarlänge wesentlich mitbenutzt, so wird die durch einen in der Wellenamplitude quadratischen Ausdruck gemessene Breite des Wellenpaketes vergrößert, statt weiter verkleinert zu werden. $\mathrm{Zu}$ der dem Komplementaritätsprinzip entsprechenden Unmöglichkeit, Ort und Impuls zugleich beliebig scharf zu messen, kommt jetzt also eine weitere Unmöglichkeit, die Messung der Ortskoordinate beliebig scharf zu machen.

\section{$\S 6$}

Es ergibt sich die Frage, ob man als Verallgemeinerung auch eine orthogonal-invariante bzw. Lorentz-invariante Multiplikation für Weltkoordinaten $x_{k}$ definieren kann. Das Axiom (2) kann in der Tat verallgemeinert werden zu einer Tensor-Beziehung:

$$
\left[x_{k}, x_{l}, x_{j} x_{h}\right]=\frac{2}{3}\left(\delta_{l k} \delta_{j h}+\delta_{l j} \delta_{k h}+\delta_{l h} \delta_{k j}\right) \text {. }
$$

Hinzufügung der im eindimensionalen Fall trivialen Beziehung

$$
\left[x_{k}, x_{l}, x_{j}\right]=0
$$

ergibt Differenzierbarkeit auch für (9).

Die weitere Grundbeziehung (5) kann verallgemeinert werden zu:

$$
\left[x_{l}, x_{l} u, v\right]=x_{l}\left[x_{k}, u, v\right]+u\left[x_{k}, x_{l}, v\right] .
$$

Die Orthogonal-Invarianz bzw. Lorentz-Invarianz von (9), (10), (11) ist ersichtlich. Im zweidimensionalen Falle ist es naheliegend, die Größe 
$w=\frac{1}{2}(x+i y) \operatorname{mit} \bar{w}=\frac{1}{2}(x-i y)$ zu benutzen. Die von $w$ allein erzeugte Unteralgebra ist dann potenzassoziativ, also trivial.

In welchem Umfang auch die gesamte aus $w$ und $\bar{w}$ erzeugte Algebra durch (9), (10), (11) festgelegt ist, kann freilich noch nicht voll übersehen werden.

\section{$\S 7$}

In I ist versucht worden, eine Klasse von Algebren, die vielleicht für physikalische Verwendung in Betracht kommen könnten, durch ein gewisses dort besprochenes Axiom zu kennzeichnen. Jedoch gehört die oben beschriebene Algebra $\hat{\mathfrak{l}}$ nicht zu derjenigen Klasse, die dieses Axiom erfüllt. (Nur Teil-Relationen des fraglichen Axioms werden durch (5) garantiert). Welche Folgerungen oder Vermutungen hieraus abzuleiten wären, bleibt vorderhand ungewiß. (Es wäre vielleicht denkbar, daß die endgültig formulierte Koordinaten-Algebra eines Elementarteilchens notwendigerweise auch den Spin zu berücksichtigen hätte.) Sowieso wird man aber im Versuche, die in I erläuterte physikalische Verwendbarkeit nichtassoziativer Algebren zu erproben, zunächst nur sehr fragmentarische Ergebnisse erhoffen können.

\section{Literatur}

1. Braun, H., u. M. Koecher: Jordan-Algebren. Berlin-Heidelberg-New York: Springer 1966.

2. Jordan, P., S. Matsushita u. H. RühaAk: Akad. Wiss. Lit. (Mainz). (Im Erscheinen.)

2 Hamburg 13, Isestr. 123 